\title{
Joppke, Christian (2021): Neoliberal Nationalism. Immigration and the Rise of the Populist Right
}

Cambridge: Cambridge University Press. 220 Seiten. $£ 19.99$

\author{
Alexander Leipold
}

Angenommen: 20. Dezember 2021 / Online publiziert: 11. Januar 2022

(C) Der/die Autor(en) 2021

Als die Alternative für Deutschland (AfD) bei der Bundestagswahl 2013 mit der Forderung nach einem Ausstieg Deutschlands aus der Europäischen Währungsunion antrat, wurde sie nicht nur von Kabarettistinnen und Kabarettisten eines irreführenden DM-Nationalismus geziehen. Dass die Partei zugleich für ein Einwanderungsrecht nach kanadischem Vorbild eintrat, rangierte unter ferner liefen im Wahlprogramm und fand in der öffentlichen Auseinandersetzung geringe Beachtung. Die damaligen Schlagzeilen waren von der Eurokrise dominiert. Wenige Beobachterinnen und Beobachter registrierten eine auf den ersten Blick merkwürdige Verquickung von neoliberaler und nationalistischer Programmatik. Die weiteren Ereignisse sind Geschichte: Die Migrationskrise ab Herbst 2015 beherrschte den innenpolitischen Diskurs und diente der AfD zur Neuausrichtung. Mit der Bundestagswahl 2017 positionierte sie sich im rechtspopulistischen Spektrum und scheint inzwischen eine dauerhafte Größe im Parteienwettbewerb, die anderen Parteien ihren Stempel aufzudrücken sucht. Das im Jahr 2019 vom Bundestag verabschiedete Fachkräfteeinwanderungsgesetz gilt auch als Reaktion auf die AfD.

Christian Joppke, Professor für Soziologie an der Universität Bern, der in den vergangenen 20 Jahren maßgebliche Arbeiten zur Politik des Multikulturalismus und zum Formwandel der Staatsbürgerschaft vorgelegt hat, weist diese Einschätzung zurück: Der Diskurs um eine restriktive, vor allem an ökonomischen Kriterien orientierte Zuwanderung habe eine wesentlich längere Vorgeschichte. Und die könne nicht auf das Erstarken rechtspopulistischer Parteien zurückgeführt werden, seien diese doch vielmehr Symptom eines langjährigen Transformationsprozesses, in dem neoliberale Denkfiguren sukzessive Eingang in Staat, Gesellschaft und damit auch das Staatsbürgerschaftsrecht gefunden haben. Vermutungen, wonach gar die liberale

Alexander Leipold (西)

Leuphana Universität Lüneburg, Lüneburg, Deutschland

E-Mail: alexander.leipold@leuphana.de 
Demokratie gefährdet sei, gingen nach Joppke fehl: Nicht der Liberalismus stehe in Rede, wohl aber der Neoliberalismus, der sich besonders in den Auswirkungen der ökonomischen Globalisierung zeige. Diese würde jedoch nicht in ökonomischen, sondern kulturellen Kategorien verhandelt und von den Rechtspopulisten auf eine Kritik an Immigration und „liberal-meritokratischen Eliten“ (S. 255) umgelenkt. Dadurch würden spezifische Unterschiede zwischen Liberalismus und Neoliberalismus verwischt. Zudem hätten viele rechtspopulistische Parteien ihren Ursprung im neoliberalen Protest gegen Steuer- und Sozialstaat.

Joppke argumentiert, dass es neben der dezidiert rechtspopulistischen Kritik eine seit Jahrzehnten gewachsene nationalistische Immigrationspolitik gibt, die gewünschte von ungewünschter Zuwanderung unterscheide. Diese Politik selektiere nicht nach ethnischen, sondern ökonomischen Erwägungen, beurteile nicht entlang von Kollektivmerkmalen, sondern individueller Fähigkeiten. Ihr immanenter Nationalismus sei kein Gegensatz zur liberalen Demokratie, sondern als Fortschritt gegenüber atavistischen Nationalismen des frühen 20. Jahrhunderts zu betrachten. Demokratische Rechtsstaaten müssten gemeinsam vereinbartes Recht nach innen wie außen durchsetzen können, was in der Konsequenz auch die legitime Ausgrenzung impliziere. Nur so könnten sie ein Mindestmaß an Teilhaberechten für die Inhaberinnen und Inhaber der Staatsbürgerschaft überhaupt aufrechterhalten. Diese Rückbindung des Einwanderungsrechts an den liberalen Konstitutionalismus sei dem Autor zufolge elementar, um der rechtspopulistischen Polemik gegen den ,kulturellen Liberalismus“ (S. 260) den Wind aus den Segeln nehmen zu können und die Debatte nicht auf ein Für und Wider von politischer Korrektheit und Identitätspolitik zu verkürzen. Letztere sei mit den Prinzipien des liberalen Konstitutionalismus unvereinbar, da sie neue Sprachverbote aufstelle (S. 261).

Praktisch realisiert würde die selektive Zuwanderung durch das Prinzip der ,,verdienten Staatsbürgerschaft“ (,,earned citizenship“, S. xi). Die Staatsbürgerschaft werde darin zum verknappten Gut, gekoppelt an einen permanenten Leistungsnachweis der Immigrierenden, während die verbliebenen sozialstaatlichen Leistungen für ihre Inhaberinnen und Inhaber konsequent beschnitten und entwertet würden. Ein Umstand, der nach Joppke für die meisten OECD-Staaten Bestand habe. So verweist der Autor die Vorstellung eines fortschrittlichen kanadischen Einwanderungsrechts in die Geschichtsbücher, da unter der Regierung Harper die Kosten für den Erwerb der Staatsbürgerschaft prohibitiv erhöht und um umfassende Nachweispflichten erweitert wurden. Kanada habe dadurch mit Großbritannien und Australien gleichgezogen. Vorreiter der Nutzenorientierung war aber Deutschland mit seiner letztlich gescheiterten Green-Card-Initiative aus dem Jahr 2000 (S. 79). Für die Rechtspopulisten sei diese Form der „Kostenorientierung“ anschlussfähig, da auch sie Zuwanderung nicht vollständig unterbinden wollten. Das Konzept der verdienten Staatsbürgerschaft sei neoliberal und nationalistisch zugleich, ,most importantly its rhetoric of ,responsibilizing" the individual“" (S. 5).

Verstehbar sei dies nur vor dem Hintergrund der Neoliberalisierung des Sozialstaates seit den 1980er-Jahren, wodurch Rechte zu Privilegien umdefiniert und (materielle) Leistungen beschnitten wurden. Damit sei das Konzept einer die Klassengegensätze überwindenden Staatsbürgerschaft im Sinne T. H. Marshalls verkehrt worden. Im permanenten Wettbewerbsstaat würden ,eingeborene“ Staatsbürgerinnen 
und Staatsbürger und außenstehende Anwärterinnen und Anwärter zu Konkurrenten um die verbliebenen Kollektivgüter. Joppke leitet dies von Friedrich Hayeks Mitte der 1970er-Jahren vorgetragenen Angriff auf den Begriff der sozialen Gerechtigkeit ab: Hayek zufolge sei soziale Gerechtigkeit eine „Illusion“ gutmeinender Planerinnen und Bürokraten aller Parteien, die nicht verstehen, dass nur der Markt organisieren könne. Eingriffe in das Privateigentum, ob durch Steuern oder Tarifverträge, würden politische Freiheit - das alleinige Gütekriterium des Neoliberalismus beschränken. Wie Joppke in einem anschließenden Parforceritt durch die Literatur zu Parteienforschung und Politischer Ökonomie des demokratischen Kapitalismus zeigt, ist dieser Glaubenssatz mit Ende des Ost-West-Konflikts auch von sozialdemokratischen Parteien aufgegriffen worden, wodurch die frühere Überzeugung der demokratischen Planbarkeit von Gesellschaft auf ein Zerrbild reduziert wurde.

Ausgehend von der Tatsache einer restriktiven Einwanderungspolitik, die Erwünschte von Unerwünschten unterscheidet, widmet sich Joppke anschließend der Frage, wie sich das Erstarken des Rechtspopulismus in Großbritannien, den USA und Deutschland auf die Zuwanderungspolitik auswirkt. Am Beispiel des BrexitReferendums legt er dar, dass sich Befürworter des EU-Ausstiegs vor allem an der Arbeitnehmerfreizügigkeit innerhalb der Europäischen Union rieben. Großbritannien, dass nach der EU-Osterweiterung überproportional viele Arbeitskräfte aus Osteuropa angezogen habe, hätte mit dem Brexit daher vor allem eine aus dem Ruder geratene Zuwanderung zu begrenzen gesucht (S. 103). Auf die britische Migrationspolitik und die gezielte Anwerbung von Hochqualifizierten werde dies nach Joppke indes keinen Einfluss haben. Die Lösung von der EU bedeute nur eine weitere Öffnung für Arbeitskräfte aus Drittstaaten - diesmal ohne Zutun aus Brüssel. Die USA hätten mit der Wahl Donald Trumps stattdessen einen vorgeblichen ,,antipopulistischen Konsens“ (Gary Freeman) in der Einwanderungspolitik aufgelöst. Doch die reaktionäre Rhetorik Trumps und die anschließenden neuen Grenzziehungen in Form von Mauerbau und „muslim ban“ kamen ohne zusätzliche Gesetzgebung aus. Trump hätte den schon von Bill Clinton beschrittenen Weg von Workfare und Armenbestrafung noch verschärft (S. 121). Dass es nicht zu weiteren Exzessen kam, so Joppke, war dann vor allem dem föderativen Staatsaufbau und den Gerichten zu verdanken, die einem populistischen Durchregieren auf bundesstaatlicher Ebene Schranken setzten. Man mag ergänzen, dass dieser antipopulistische Konsens dann auch nicht mehr als eine Momentaufnahme gewesen sein kann und seine normative Bindungskraft nach Jahrzehnten der Globalisierung zur Phrase geworden ist.

Anders in Deutschland, wo mit den Hartzreformen der Regierung Schröder der europaweit größte Niedriglohnsektor etabliert wurde. Die Regierung beabsichtigte eine Verringerung der seit der Wiedervereinigung anhaltenden Massenarbeitslosigkeit. Der Beschluss der Reformen im Vorfeld der EU-Osterweiterung war Joppke zufolge kein Zufall (S. 29). Der Hartz-IV-Regelsatz von 650 Euro entsprach etwa dem Durchschnittslohn in Polen und Tschechien und verringerte Anreize zur Arbeitsmigration in den Dienstleistungsberufen, während die noch tarifvertraglich gesicherten Arbeitsplätze in verarbeitender Industrie und boomender Exportwirtschaft eine gewisse „Schutzzone“ gebildet hätten. Diese wirkte auch während der Eurokrise fort und ermöglichte Deutschland das Festhalten an seiner Einwanderungspolitik, auch nach Erstarken der AfD. Die Öffnung der Balkanroute im Herbst 
2015 hatte dann möglicherweise die populistischen Wahlerfolge in Großbritannien und den USA ungewollt angefacht, indem es Ängste mobilisierte, die sich trefflich politisch ausschlachten ließen (S. 136-137). Der deutsche Fall sei auch deswegen besonders, weil der im Zuge der Migrationskrise entstandene Diskurs über etwaige Restriktionen des deutschen Zuwanderungsrechts und daran geknüpfte Forderungen nach offenen Grenzen den Fakten nicht standhalte. Das 2019 beschlossene „Rückführungsgesetz“, so Joppke, muss gerade von Anhängerinnen und Anhängern des politischen Liberalismus als Mittel zur Sicherung des demokratischen Rechtstaats und der individuellen Freiheitsrechte aller Gesellschaftsmitglieder verstanden werden. Zudem könnten die dadurch ermöglichten Abschiebungen in der Praxis auch weiterhin unterlaufen werden, seien also nach Joppke gar nicht so restriktiv, wie der moralisch aufgeladene Disput suggerieren würde. Es handele sich vielmehr um eine ,necessary flip side of the open-border policy for refugees, from which the German government, in principle, has never moved away“ (S. 274-275).

Joppke gelangt zu einem unpolitischen, wenn auch nüchternen Ergebnis: Zwar stünden die liberalen Demokratien nicht zur Disposition, doch werde die nutzenorientierte Zuwanderungspolitik alternativlos bleiben, sollen die Sozialstaaten nicht überlastet werden. Denn die Entwertung der Staatsbürgerschaft werde angesichts uneingeschränkter Austeritätspolitik fortdauern. Er beschließt sein Buch mit einer vom britischen Intellektuellen David Goodhart aufgebrachten Dichotomie von Solidarität und Diversität (S. 283). Solidarität, also die Gewähr von Teilhaberechten in einer abgrenzbaren Gemeinschaft, könne in offenen Gesellschaften und unter der Bedingung der Personenfreizügigkeit nicht aufrechterhalten werden, man müsse diese im Zweifel opfern. Der hierdurch hervorgerufene Konflikt zwischen Gewinnern und Verlierern der Offenheit sei aber insgesamt nicht bedeutend genug, um eine Abkehr von der heute mehrheitlich höherrangig bewerteten Diversität der Gesellschaften zuzulassen: „Neoliberal-cum-nationalist restrictions could only affect this opening at the margins“ (S. 283). Diese Einschätzung wirft unweigerlich Folgefragen auf, denn das Ausmaß der Krisenwahrnehmung ist eine veränderliche Größe, sodass kein abschließendes Urteil gefällt werden kann. Es nimmt überdies der zuvor so ausführlich dargelegten Kritik des Neoliberalismus den Stachel, indem sie die sehr realen sozialen Schließungsprozesse in und zwischen den Staaten zu akzeptieren scheint. Die Verlierer der Offenheit - hüben wie drüben - werden dies kaum für zufriedenstellend erachten können. Für sozialdemokratische Parteien ist diese Diagnose indes fatal, da sie konstitutive politische Grundüberzeugungen über Bord wirft und für ihren andauernden elektoralen Niedergang nichts Gutes verheißt.

Funding Open Access funding enabled and organized by Projekt DEAL.

Open Access Dieser Artikel wird unter der Creative Commons Namensnennung 4.0 International Lizenz veröffentlicht, welche die Nutzung, Vervielfältigung, Bearbeitung, Verbreitung und Wiedergabe in jeglichem Medium und Format erlaubt, sofern Sie den/die ursprünglichen Autor(en) und die Quelle ordnungsgemäß nennen, einen Link zur Creative Commons Lizenz beifügen und angeben, ob Änderungen vorgenommen wurden.

Die in diesem Artikel enthaltenen Bilder und sonstiges Drittmaterial unterliegen ebenfalls der genannten Creative Commons Lizenz, sofern sich aus der Abbildungslegende nichts anderes ergibt. Sofern das betreffende Material nicht unter der genannten Creative Commons Lizenz steht und die betreffende Handlung 
nicht nach gesetzlichen Vorschriften erlaubt ist, ist für die oben aufgeführten Weiterverwendungen des Materials die Einwilligung des jeweiligen Rechteinhabers einzuholen.

Weitere Details zur Lizenz entnehmen Sie bitte der Lizenzinformation auf http://creativecommons.org/ licenses/by/4.0/deed.de. 